\title{
A search for TiO in the optical high-resolution transmission spectrum of HD 209458b: Hindrance due to inaccuracies in the line database
}

\author{
H. J. Hoeijmakers ${ }^{1}$, R. J. de Kok ${ }^{1,2}$, I. A. G. Snellen ${ }^{1}$, M. Brogi ${ }^{1, \star}$, J. L. Birkby ${ }^{1,3, \star \star}$, and H. Schwarz ${ }^{1}$ \\ ${ }^{1}$ Leiden Observatory, University of Leiden, Niels Bohrweg 2, 2333 CA Leiden, The Netherlands \\ e-mail: hoeijmakers@strw.leidenuniv.nl \\ 2 SRON, Netherlands Institute for Space Research, Sorbonnelaan 2, 3584 CA Utrecht, The Netherlands \\ ${ }^{3}$ Harvard-Smithsonian Center for Astrophysics, 60 Garden Street, Cambridge, MA 02138, USA
}

Received 12 August 2014 / Accepted 25 October 2014

\section{ABSTRACT}

\begin{abstract}
Context. The spectral signature of an exoplanet can be separated from the spectrum of its host star using high-resolution spectroscopy. During these observations, the radial component of the planet's orbital velocity changes, resulting in a significant Doppler shift that allows its spectral features to be extracted.

Aims. In this work, we aim to detect TiO in the optical transmission spectrum of HD 209458b. Gaseous TiO has been suggested as the cause of the thermal inversion layer invoked to explain the dayside spectrum of this planet.

Methods. We used archival data from the $8.2 \mathrm{~m}$ Subaru telescope taken with the High Dispersion Spectrograph of a transit of HD 209458b in 2002. We created model transmission spectra that include absorption by TiO, and cross-correlated them with the residual spectral data after removal of the dominating stellar absorption features. We subsequently co-added the correlation signal in time, taking the change in Doppler shift due to the orbit of the planet into account.

Results. We detect no significant cross-correlation signal due to $\mathrm{TiO}$, though artificial injection of our template spectra into the data indicates a sensitivity down to a volume-mixing ratio of $\sim 10^{-10}$. However, cross-correlating the template spectra with a HARPS spectrum of Barnard's star yields only a weak wavelength-dependent correlation, even though Barnard's star is an M4V dwarf that exhibits clear $\mathrm{TiO}$ absorption. We infer that the $\mathrm{TiO}$ line list poorly matches the real positions of $\mathrm{TiO}$ lines at spectral resolutions of $\sim 100000$. Similar line lists are also used in the PHOENIX and Kurucz stellar atmosphere suites and we show that their synthetic M-dwarf spectra also correlate poorly with the HARPS spectra of Barnard's star and five other M dwarfs. We conclude that the lack of an accurate $\mathrm{TiO}$ line list is currently critically hampering this high-resolution retrieval technique.
\end{abstract}

Key words. line: identification - molecular data - planets and satellites: atmospheres - methods: observational

\section{Introduction}

Recently, carbon monoxide has been identified in the transmission spectrum of the exoplanet HD 209458b (Snellen et al. 2010). During transit, the radial velocity of the hot Jupiter changes by a few tens of $\mathrm{km} \mathrm{s}^{-1}$, resulting in a changing Doppler shift that is measurable at a resolution of $R \sim 10^{5}$. This time variation of the planet's spectral signature provides a powerful tool to distinguish between the planet and the overwhelmingly bright host star, because the absorption features in the stellar spectrum are quasi-invariant on these time scales (see Fig. 1). Furthermore, because the orbital period of the planet is well known, its radial velocity is known at all times, and so the Doppler shift of the planets' absorption features can be targeted specifically.

This technique has also been successfully applied in other parts of the orbit, where direct thermal emission of the planet is probed. Absorption in the dayside spectra of both transiting and non-transiting hot Jupiters has been observed: $\mathrm{CO}$ and $\mathrm{H}_{2} \mathrm{O}$ absorption in the atmospheres of $\tau$ Boötes b, HD $189733 \mathrm{~b}$, 51 Peg b and HD 179733 b (Birkby et al. 2013; Brogi et al. 2012, 2013, 2014; de Kok et al. 2013; Lockwood et al. 2014; Rodler et al. 2013). In the case of the non-transiting planets, this

$\star$ NASA Hubble Fellow.

$\star \star$ NASA Sagan Fellow. has allowed for their previously unknown orbital inclinations to be determined as $44.5^{\circ} \pm 1.5^{\circ}$ for $\tau$ Boötes b (Brogi et al. 2012), which is consistent with the measurement of Rodler et al. (2012), $79.6^{\circ}-82.2^{\circ}$ for 51 Peg b (Brogi et al. 2013), and $67.7^{\circ} \pm 4.3^{\circ}$ for HD 179733 b (Brogi et al. 2014), which in turn lead to a determination of their masses.

HD 209458b is a well-studied exoplanet. It orbits a solartype star once every 3.5 days and is located $47 \mathrm{pc}$ from the Sun (see Table 1 for the physical properties of the system). Using the Spitzer Space Telescope Knutson et al. (2008) found evidence for $\mathrm{H}_{2} \mathrm{O}$ emission in the IRAC bands at $4.5 \mu \mathrm{m}$ and $5.8 \mu \mathrm{m}$, which is indicative of a thermal inversion layer in the atmosphere of the planet. A temperature inversion is caused by the absorption of incident starlight in a high-altitude layer of the atmosphere. On Earth, solar UV radiation is absorbed in the oxygenozone cycle, effectively absorbing all sunlight short of $350 \mathrm{~nm}$, heating the atmosphere and forming the stratosphere between 10 and $50 \mathrm{~km}$ altitude (see e.g. Portmann \& Solomon 2007, and references therein). In the atmospheres of hot Jupiters, similar processes may occur, albeit in different chemical environments.

A number of compounds have been proposed as capable of causing the inversion layer in the atmosphere of HD 209458b, one of which is TiO (Hubeny et al. 2003; Burrows et al. 2007), a diatomic molecule that is known to cause major absorption features throughout the optical and near-infrared spectra of 


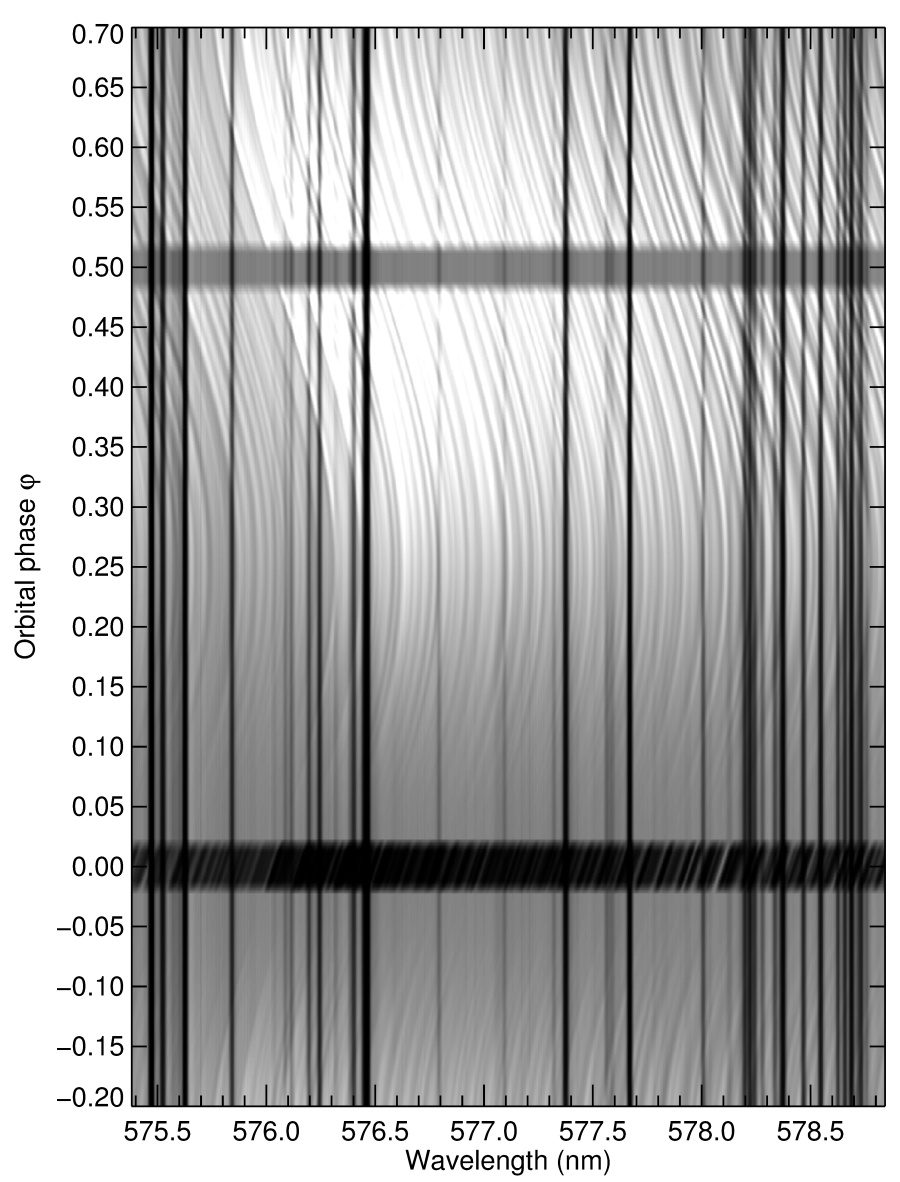

Fig. 1. Toy model of the phase-dependent Doppler shift of TiO lines along the orbit of HD 209458b. The white curves represent TiOemission features owing to the inversion layer (greatly enhanced for visual purposes). During transit around $\phi=0$, TiO produces slanted absorption lines (black). The black vertical lines are stellar absorption lines, which are stable in time. This difference in the behaviour of stellar and planetary features provides a means of contrast between star and planet.

$\mathrm{M}$ dwarfs. An atmosphere containing a strong optical absorber like TiO would furthermore be consistent with HD 209458b's low albedo (Rowe et al. 2006). Désert et al. (2008) report a tentative detection of $\mathrm{TiO}$ absorption in the transmission spectrum of HD 209458b, though more recent broadband studies seem to support a lack of $\mathrm{TiO}$ absorption in the transmission spectra of other hot Jupiters (see e.g. Huitson et al. 2013; Sing et al. 2013; Gibson et al. 2013; Bento et al. 2014). Also, models of hot-Jupiter atmospheres suggest that maintaining a significant gaseous $\mathrm{TiO}$ concentration at high altitudes is difficult because of the condensation of $\mathrm{TiO}$ either at depth or on the night side (Spiegel et al. 2009; Burrows \& Orton 2009; Fortney et al. 2010; Parmentier et al. 2013). More recent observations of the day-side emission spectrum of HD 209458b, call the existence of the inversion layer into question (Zellem et al. 2014; Diamond-Lowe et al. 2014). Interestingly, Stevenson et al. (2014) observed molecular absorption features in the near-infrared dayside spectrum of Wasp-12b, possibly attributable to TiO. This contrasts with the optical transmission spectra of the same planet, as put forth by Sing et al. (2013). Thus, it is as yet unclear whether TiO is an important chemical component of hot-Jupiter atmospheres.

In this paper we apply a cross-correlation-based retrieval method to search for $\mathrm{TiO}$ in the transmission spectrum of
Table 1. Properties of HD 209458 (upper part) and HD 209458b (lower part), adopted from Torres et al. (2008).

\begin{tabular}{|c|c|c|}
\hline Parameter & Symbol & Value \\
\hline Visible magnitude & $V$ & $7.67 \pm 0.01$ \\
\hline Distance (pc) & $d$ & $47.4 \pm 1.6$ \\
\hline Effective temperature $(\mathrm{K})$ & $T_{\text {eff }}$ & $6065 \pm 50$ \\
\hline Luminosity $\left(L_{\odot}\right)$ & $L_{*}$ & $1.622_{-0.10}^{+0.097}$ \\
\hline Mass $\left(M_{\odot}\right)$ & $M_{*}$ & $1.119 \pm 0.033$ \\
\hline Radius $\left(R_{\odot}\right)$ & $R_{*}$ & $1.155^{+0.014}$ \\
\hline Systemic velocity $\left(\mathrm{km} \mathrm{s}^{-1}\right)^{a}$ & & $-14.7652 \pm 0.0016$ \\
\hline Metallicity (dex) & {$[\mathrm{F} / \mathrm{H}]$} & $0.00 \pm 0.05$ \\
\hline Age (Gyr) & & $3.1_{-0.7}^{+0.8}$ \\
\hline Orbital period (days) ${ }^{b}$ & $P$ & $\begin{array}{l}3.52474859 \\
\pm 0.00000038\end{array}$ \\
\hline axis $(\mathrm{AU})$ & $a$ & $0.04707_{-0.00047}^{+0.00046}$ \\
\hline (deg) & $i$ & $86.71 \pm 0.05$ \\
\hline Eccentricity $^{c}$ & $e \cos \omega$ & $0.00004 \pm 0.00033$ \\
\hline Impact parameter & $b$ & $0.507 \pm 0.005$ \\
\hline Transit central time $(\mathrm{HJD})^{b}$ & $t_{c}$ & $\begin{array}{l}2,452,826.628521 \\
\pm 0.000087\end{array}$ \\
\hline Transit duration $(\min )^{d}$ & $t_{T}$ & $183.89 \pm 3.17$ \\
\hline $\operatorname{Mass}\left(M_{J}\right)$ & $M_{p}$ & $0.685_{-0.014}^{+0.015}$ \\
\hline Radius $\left(R_{J}\right)$ & $R_{p}$ & $1.359_{-0.019}^{+0.016}$ \\
\hline Density $\left(\mathrm{g} \mathrm{cm}^{-3}\right)$ & $\rho_{p}$ & $0.338_{-0.014}^{+0.016}$ \\
\hline Equilibrium temperature $(\mathrm{K})$ & $T_{\text {eq }}$ & $1449 \pm 12$ \\
\hline
\end{tabular}

Notes. ${ }^{(a)}$ Adopted from Mazeh et al. (2000); ${ }^{(b)}$ adopted from Knutson et al. (2007); ${ }^{(c)}$ adopted from Crossfield et al. (2012); ${ }^{(d)}$ adopted from the Exoplanet Orbit Database (Wright et al. 2011).

HD 209458b at high spectral resolution. Section 2 describes our dataset and the application of the method. Section 3 discusses our results, followed by our conclusions in Sect. 4 .

\section{Observations and data reduction}

\subsection{Subaru data of HD 209458}

The data used in this analysis were taken on October 25, 2002 using the High Dispersion Spectrograph (HDS) on the Subaru $8.2 \mathrm{~m}$ telescope. The observations were performed by Narita et al. (2005) and were originally aimed at detecting absorption of sodium and number of other atomic species in the transmission spectrum of HD 209458b. These were later used by Snellen et al. (2008) who did indeed detect sodium. The data consist of 31 exposures with a mean exposure time of $500 \mathrm{~s}$ and cover one $3 \mathrm{~h}$ transit, plus $1.5 \mathrm{~h}$ and $0.5 \mathrm{~h}$ of baseline before and after, respectively. The spectrum is observed over 20 echelle orders, covering a wavelength range between $554 \mathrm{~nm}$ and $682 \mathrm{~nm}$ at a spectral resolution of $R \sim 45000$ (6.7 $\mathrm{km} \mathrm{s}^{-1}$ resolution). Each spectral order is sampled at $0.9 \mathrm{~km} \mathrm{~s}^{-1}$ per pixel over 4100 pixels. The basic data reduction (such as bias subtraction, flat fielding and 1D spectral extraction) were performed by Snellen et al. (2008) and are described therein in detail.

\subsection{HARPS data of M dwarfs}

Our analysis relies on cross-correlating the transmission spectra of HD 209458b with high-resolution template spectra of TiO-bearing models of HD 209458b's absorption spectrum which rely on line lists published by Freedman et al. (2008, see Sects. 2.3 and 2.4). The optical spectra of $M$ dwarfs are 
M-dwarf templates

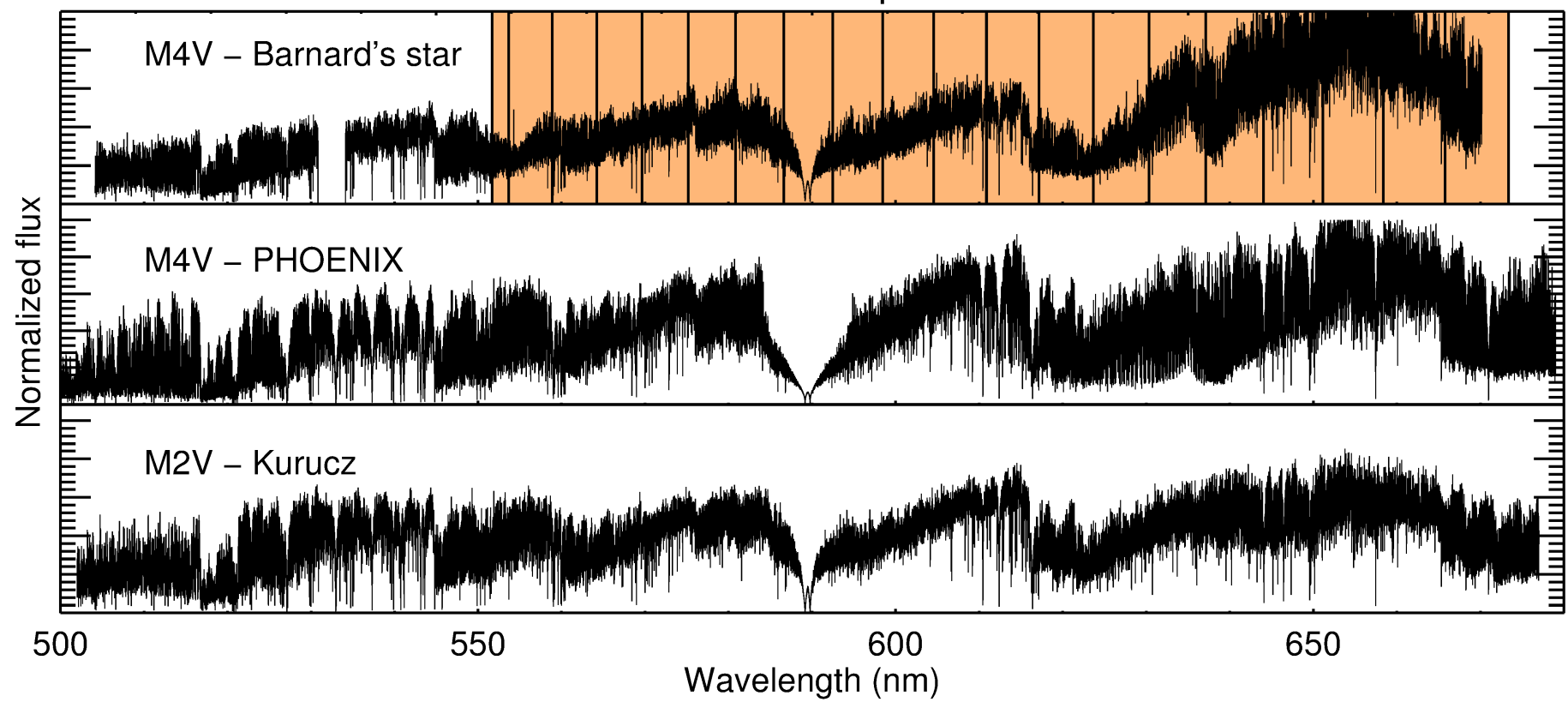

Fig. 2. Top panel: archival HARPS spectrum of Barnard's star at a resolution of $R=115000$. Middle panel: a PHOENIX model of a generic M4V dwarf. Bottom panel: Kurucz model of Gliese 411, a standard M2V dwarf. These three templates contain strong broadband features, which are removed by applying a high-pass filter later in the analysis (not shown). The orange region in the top panel covers the approximate extent of the spectral orders of the HDS Subaru dataset, which are delimited by the solid vertical lines.

Table 2. Overview of the archival M-dwarf spectra used in this work, obtained using the HARPS-south instrument.

\begin{tabular}{llll}
\hline \hline Name & Spectral type & PID (ESO) & PI \\
\hline Proxima Centauri & M6.0V & 183.C-0437(A) & Bonfils \\
GJ402 & M5.0V & 183.C-0437(A) & Bonfils \\
GJ9066 & M4.5V & 072.C-0488(E) & Mayor \\
GJ876 & M5.0V & 183.C-0437(A) & Bonfils \\
Barnard's star & M4.0V & 072.C-0488(E) & Mayor \\
\hline
\end{tabular}

dominated by $\mathrm{TiO}$ absorption features and are therefore natural benchmarks against which we tested the accuracy of these TiO templates. Many nearby M-dwarfs have been observed extensively at high spectral resolution in order to detect the radial velocity signature induced by potential orbiting companions. We downloaded high-resolution spectra of five different $M$ dwarfs from the ESO data archive. These spectra were obtained by the HARPS spectrograph at ESO's $3.6 \mathrm{~m}$ La Silla telescope, between $500 \mathrm{~nm}$ and $691 \mathrm{~nm}$ at resolutions of $R=115000$. Information about the five stars is shown in Table 2. Barnard's star is used throughout this work as the primary example, and is shown in the top panel of Fig. 2 before the removal of all broadband features using a high-pass filter (the spectra of the other $\mathrm{M}$ dwarfs were filtered in the same way), and after correcting for its radial velocity of $-110.51 \mathrm{~km} \mathrm{~s}^{-1}$ (Nidever et al. 2002).

\subsection{Post-processing and cross-correlation}

The analysis of the high dispersion transmission spectra of HD 209458b differs at several points from that of infrared data used in previous high-dispersion spectroscopy studies by our group (see Birkby et al. 2013; Brogi et al. 2012, 2013, 2014; de Kok et al. 2013), which are dominated by time-dependent telluric absorption features, mainly due to variations in airmass.
In the optical, the atmosphere presents no significant absorption features except in some specific wavelength ranges. Rather, the spectrum is dominated by stellar absorption lines. Assuming that the stellar spectrum is stable during the transit, it can be removed by dividing each of the individual spectra in the time series with the time-averaged stellar spectrum. In principle, this operation does not affect the planet signal because it significantly shifts in wavelength during the observations owing to the change in the radial component of the orbital velocity during transit (from $-15 \mathrm{~km} \mathrm{~s}^{-1}$ to $+15 \mathrm{~km} \mathrm{~s}^{-1}$ ).

The full sequence of processing steps is summarized below. Intermediate data products are shown in Fig. 3.

1. Broadband removal and normalization: to remove strong broadband components such as the stellar continuum and the spectrograph's blaze function, the brightest exposure of each spectral order was divided into 50 wavelength regions. A seventh order polynomial was fit through the maximum of each part to obtain the continuum level. This fit was removed from all other exposures through division, removing the strongest broadband features. Subsequently, the continuum of each of the exposures was obtained by dividing each exposure into the same 50 regions as above and again finding the maximum of each part. These continua were interpolated, and removed through division. This acts to remove residual broad-band variations not removed by the seventh-order polynomial fit, and normalizes the continuum of each exposure to unity. At this stage there were still significant residual broad-band variations, which were removed at a later stage (see step 7).

2. Alignment of spectra: the spectra slowly drift in wavelength because of both the change in radial velocity of the observatory during the observations and instrumental instability. These misalignments are approximately a pixel and would adversely affect the removal of the time-average stellar 


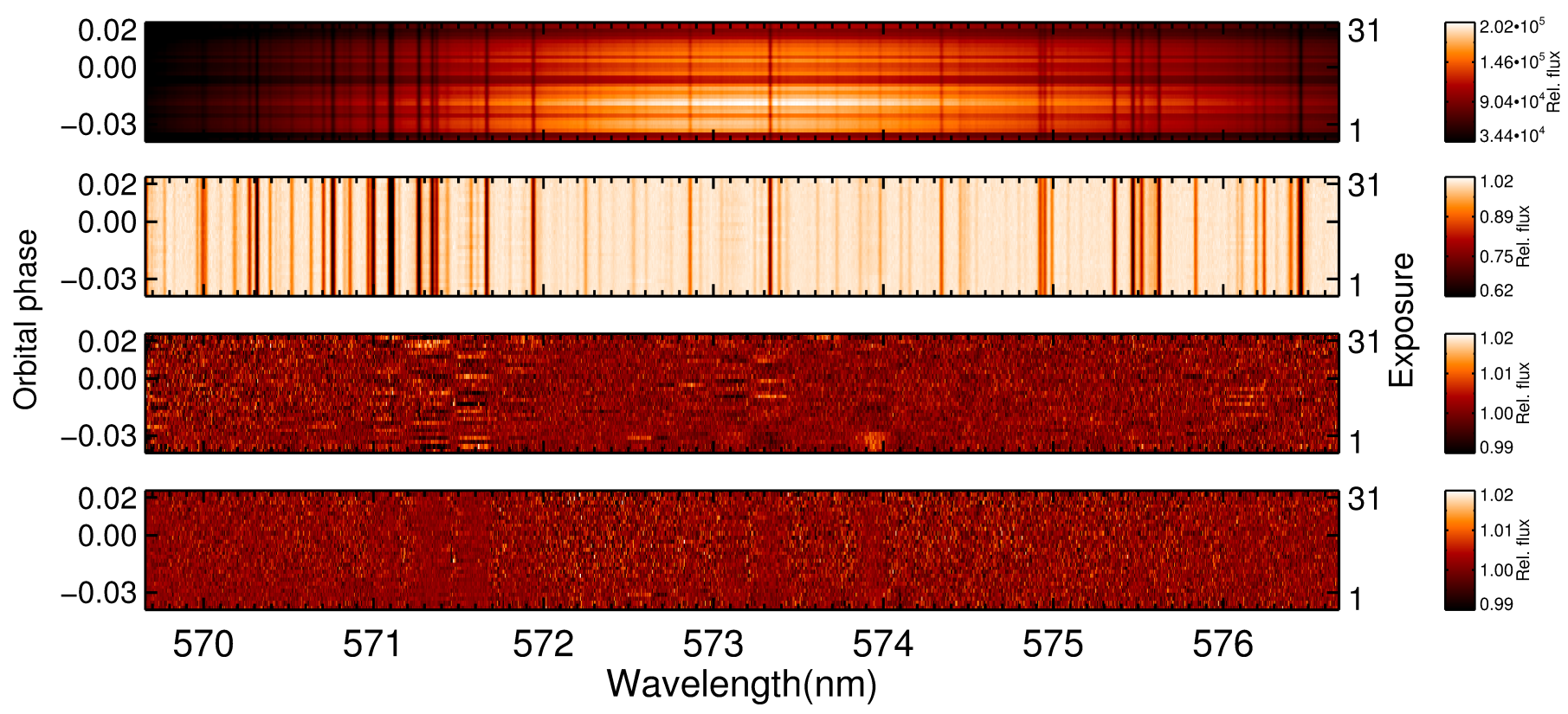

Fig. 3. Major post processing steps of echelle order 17 before cross-correlation. The top panel: shows the initial state of the spectral order, arranged such that all exposures in this order are stacked vertically. The result of the removal of the blaze pattern and the alignment of the individual exposures (which cannot be seen by eye because the misalignments are on a sub-pixel level) is shown in the second panel. Third panel: residuals after dividing through the time-averaged spectrum (i.e. the average of the spectrum obtained by averaging each column in the second panel). Fourth panel: same residuals after removing $4 \sigma$ outliers, removing latent broadband features and weighing down noisy columns. The average standard deviation of the residuals at this stage is $0.45 \%$. Only the lower two panels are scaled to the same colour range.

spectrum if left untreated. In each spectral order, a dozen strong stellar absorption lines were identified by eye. To these lines in each of the 31 exposures, Gaussian profiles were fitted to obtain the position of each line centre. A linear fit through the misalignments of the different absorption lines was used to align the spectra to a common reference frame. (A higher order fit was not warranted because the scatter in the determination of the line centroids was too high to identify higher order components.)

3. Wavelength solution: since HD 209458 is a close solar analogue, we matched our time-averaged spectrum (see next step) of the HD 209458 system to a model solar spectrum (adopted from the Kurucz stellar atmosphere atlas) in a stepwise fashion. The strongest stellar absorption lines were identified and matched by eye. This produced a crude wavelength solution that was subsequently used to identify 30-40 less prominent lines in each order. We subsequently fitted Gaussian profiles to the cores of these lines in both the data and the solar template, producing a wavelength solution with a standard deviation of $1.7 \times 10^{-3} \mathrm{~nm} \pm 0.7 \times 10^{-3}$, corresponding to $0.83 \pm 0.36 \mathrm{~km} \mathrm{~s}^{-1}$ or $\sim 12 \%$ of the spectral resolution. Because the solar spectrum is synthesized at rest, the wavelength solution is calibrated to the rest frame of HD 209458, so the mean radial velocity with respect to the observatory on Earth is automatically corrected for. Any time-dependent Doppler shifts had already been corrected in step 2. Therefore, each exposure is now in the rest frame of the host star.

4. Removal of stellar spectrum: each pixel in each spectral order was time-averaged by taking the median of the aligned spectra, which corresponds to taking the median of each of the columns of the array in panel 2 of Fig. 3. This average contains all time-constant stellar features, but only a small part of the time-varying planet signal, since it is shifted to a different column in each exposure. It is removed from each exposure through division. The width of planetary absorption lines is limited to $6.7 \mathrm{~km} \mathrm{~s}^{-1}$, which is the resolution of the instrument. Between exposures, the planet signal is shifted by $\sim 1.8 \mathrm{~km} \mathrm{~s}^{-1}$. Therefore, the planet signal is present in no more than four exposures per pixel column. These four exposures are part of a median with 27 other exposures that do not contain the planet signal. Therefore, we expect that up to $10 \%-15 \%$ of the planet signal is removed along with the median stellar spectrum. This is not important for the rest of the analysis, because we measure the significance of any $\mathrm{TiO}$ detection by our ability to retrieve injected model spectra, which suffer in the same way from this degradation (see Sect. 3).

5. Removal of bad pixels and cosmics: the resulting residuals contain bad pixels and cosmic rays. All pixels deviating by more than $4 \sigma$ from the mean were reset to the mean, removing all significant bad pixels and cosmic rays. As a result, $0.21 \%$ of all pixel values were affected.

6. Normalization by signal to noise: the noise in the residual spectrum varies with pixel position owing to the presence of deep stellar absorption lines up until step 4 . We divide each pixel in the residual spectrum by the variance of that pixel value (variance of each column of panel 3, Fig. 2.3) to weigh down such pixels. Low $\mathrm{S} / \mathrm{R}$ regions would otherwise add a significant amount of noise to the cross-correlation function.

7. Suppression of broadband residuals: the residuals feature broadband variations due to imperfect removal of instrumental effects and broad stellar lines. Latent broadband variations were characterized by smoothing each exposure with a Gaussian with a width of $40 \mathrm{px}\left(36 \mathrm{~km} \mathrm{~s}^{-1}\right)$ and were divided out of the data. The last panel of Fig. 3 shows the residual of this final processing step, before the cross-correlation is performed. 

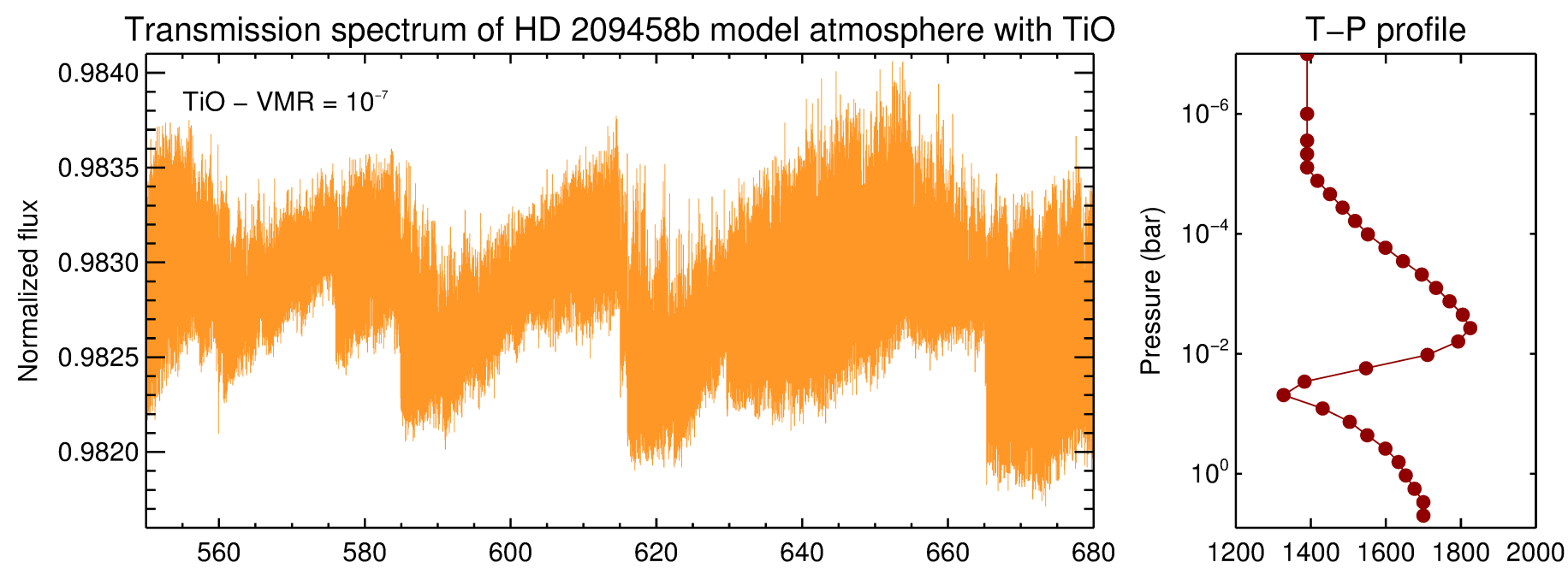

Fig. 4. Left panel: one of our model absorption spectra of HD $209458 \mathrm{~b}$ containing $\mathrm{TiO}$ at a VMR of $10^{-7}$, normalized to the brightness of the system out of transit and according to the temperature-pressure profile shown on the right, along with the corresponding altitude in units of the planet radius. Throughout this work, we employ this spectrum as a template in various cross-correlation analyses, as well as four similar templates at lower VMRs; and one template at a constant temperature of $3000 \mathrm{~K}$. Note that the form of the assumed T-P profile does not significantly impact the correlation, because the effective temperature of the star is $\sim 6065 \mathrm{~K}$.

8. Cross-correlation: the planet's potential absorption features were extracted by cross-correlating the residual spectra with template spectra of $\mathrm{TiO}$ (see Sect. 2.4) across the full wavelength range of the data, so between $\sim 554$ and $\sim 682 \mathrm{~nm}$. The templates were Doppler-shifted to velocities ranging between $\pm 150 \mathrm{~km} \mathrm{~s}^{-1}$ relative to the star, in steps of $1 \mathrm{~km} \mathrm{~s}^{-1}$ (which roughly corresponds to the sampling rate of the data, $0.9 \mathrm{~km} \mathrm{~s}^{-1}$ per pixel). At the Doppler shift equal to the radial velocity of the planet, the cross-correlation effectively coadds all of the individual absorption lines of the molecule.

\subsection{Model spectra}

For the purpose of applying the cross-correlation method to the case of $\mathrm{TiO}$ in the atmosphere of HD 209458b (see below), we generated several model transmission spectra of atmospheres containing various concentrations of $\mathrm{TiO}$, at a sampling resolution of $R=240000$. The line database stems from Freedman et al. (2008), who present a modified line list based on the one published by Schwenke (1998). The model atmosphere has a temperature-pressure profile as shown in Fig. 4, and includes $\mathrm{H}_{2}$ scattering and molecular absorption due to $\mathrm{TiO}$ with volume mixing ratios (VMR) of $10^{-7}, 10^{-8}, 10^{-9}, 10^{-10}$ and $10^{-11}$. The solar photospheric abundance of titanium is $10^{-7.1}$ (Asplund et al. 2006), so the transmission spectrum of $\mathrm{TiO}$ is modelled between $\sim 1$ and $\sim 10^{-4}$ times the solar abundance of Ti. These mixing ratios were assumed to be uniform throughout the atmosphere. As such, no chemistry, phase changes or dynamics were taken into account. The atmosphere was assumed to have a temperature inversion as displayed in the right-hand panel of Fig. 4 (adopted from Burrows et al. 2007), but because the background continuum originates in a star with an effective temperature of $6065 \mathrm{~K}$, the assumption of a particular T-P-profile does not significantly influence the template spectrum. To confirm the robustness of the template to a temperature mismatch, we also modelled a TiO-bearing atmosphere at a constant temperature of $3000 \mathrm{~K}$ and cross-correlated it with the template shown in Fig. 4. The result is shown in Fig. 6, and discussed in more detail in Sect. 3.1.
For benchmark purposes (see Sect. 2.2), we also crosscorrelated with synthetic M-dwarf spectra originating in the Kurucz and PHOENIX stellar atmosphere model suites which necessarily include models of the TiO molecule. Both models use a line list calculated by Plez (1998). The Kurucz model is a template spectrum of the M2V dwarf GL411 at a resolution of $R=100000$ (Castelli \& Kurucz 2003) and the PHOENIX model, a template of a generic M4V dwarf $\left(T_{\mathrm{eff}}=3100 \mathrm{~K}\right.$, $\log g=5.0, \mathrm{Fe} / \mathrm{H}=+0.5$, obtained from the PHOENIX online database as presented by Husser et al. 2013) at a resolution of $R=125000$. They are shown together with the HARPS spectrum of Barnard's star in Fig. 2.

\section{Results and discussion}

The left-hand panels of Fig. 5 show the correlations between the HDS spectra of HD 209458b and our TiO template at five modelled VMRs (see Sect. 2.4). The correlation coefficients are plotted as a function of the radial velocity to which the template is shifted (horizontal axis) for every individual exposure (i.e. time, vertical axis).

To assess the ability of the cross-correlation procedure to retrieve the template spectra from real data, we inject the templates into the data prior to cross-correlation using the known orbital velocity of HD 209458b, between steps 3 and 4 in Sect. 2.3, similar to Snellen et al. (2010). Prior to injection, we convolve the template with a Gaussian to match the spectral resolution of the data (45000), and convolve again with a box function to account for the widening of the planetary absorption lines due to the changing Doppler shift during the 500s exposures. This gives rise to the middle panels in Fig. 5. Injection of a TiO-bearing template at a VMR greater than $10^{-10}$ clearly produces a slanted feature, whose gradient is a measure of the orbital velocity of the planet at which the template was injected.

Given that the radial velocity of the planet is known at all times, each exposure (i.e. row in Fig. 5) can be shifted to the rest frame of the planet. This allows for co-addition of all exposures, the result of which is shown in the right-hand panels in Fig. 5. The signal-to-noise ratio $(\mathrm{S} / \mathrm{N})$ of the observed correlation peak is indicative of the significance at which the template 

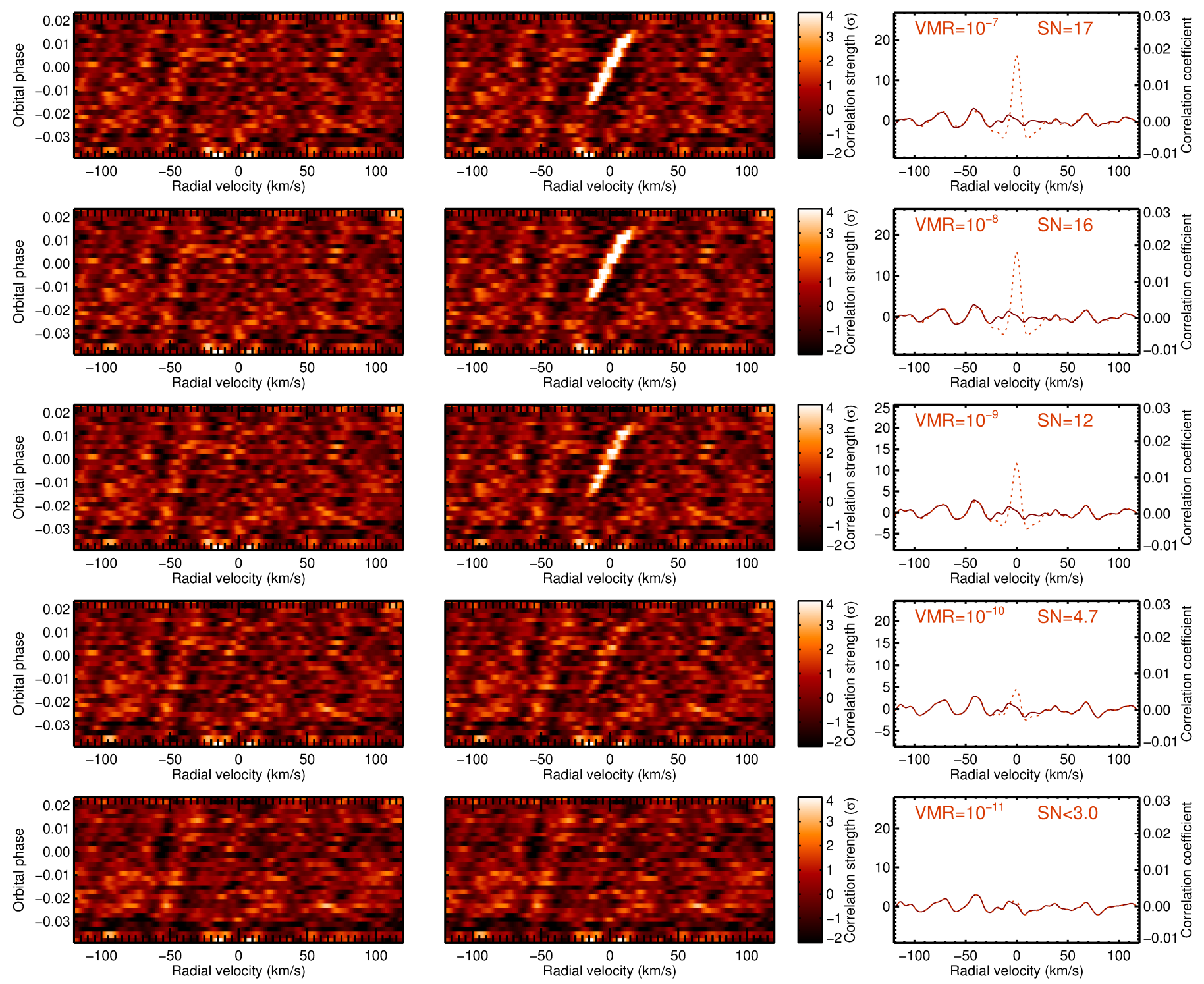

Fig. 5. Strength of the cross-correlation signature as a function of radial velocity and orbital phase. Each row of panels shows the cross-correlation with model atmospheres with different VMR's of TiO (left panels, ranging from $10^{-7}$ (top) to $10^{-11}$ (bottom) by factors of 10). The models are also injected into the data prior to cross-correlation (middle panels) to probe the sensitivity of the procedure. Co-addition of all exposures (right panels) is achieved by shifting each exposure (i.e. each row in the middle and left panels) to the rest-frame of the planet and averaging them in time. The dashed lines are the co-added correlation strength of the residuals, after having injected them with the model template with which the crosscorrelation is performed. The solid lines are the co-added correlation strengths of the residuals without prior injection of the template. The $\mathrm{S} / \mathrm{N}$ of the correlation peak is calculated by taking the peak correlation, and dividing it through the standard deviation of the cross-correlation response, excluding a range of $\pm 20 \mathrm{~km} \mathrm{~s}^{-1}$ in which the correlation peak itself resides. Clearly, the presence of TiO would be convincingly retrieved at the $\mathrm{ppb}$ level, provided that the line database is accurate.

would be retrieved, had it been identically present in the residuals. Evidently, the peak correlation strength decreases with decreasing VMR, hence the VMR at which the correlation peaks at the $3 \sigma$ level, which we consider to be the theoretical limiting VMR at which $\mathrm{TiO}$ would be retrievable by applying the current analysis on this data.

It is clear from the left-hand panels of Fig. 5 that the residuals do not correlate with our $\mathrm{TiO}$ templates. This non-detection is significant down to VMRs of $10^{-10}$, as shown by the successful retrieval of our templates after having artificially injected them into the data. This sensitivity limit corresponds to $10^{-3}$ times the solar abundance of Ti (Asplund et al. 2006). The models of the atmosphere of HD $209458 \mathrm{~b}$ are greatly simplified approximations of the real physical environment. The assumption of a uniform VMR across the entire atmosphere is especially questionable, because the atmosphere is not expected to be homogeneous. However, by extending the absorbing $\mathrm{TiO}$ layer uniformly throughout the atmosphere, the TiO absorption at that VMR is maximized. The limiting VMR of $10^{-10}$ is therefore a true lower limit: If TiO were only present at a VMR of $10^{-10}$ in parts of the atmosphere, it would not be retrieved in this analysis.

Before invoking a plethora of reasons for this non-detection of TiO that include hazes, obscuring clouds, screening by other species, cold-traps or destructive chemistry, it should be noted that the sensitivity of the cross-correlation procedure critically depends on the accuracy of the line positions in the template 


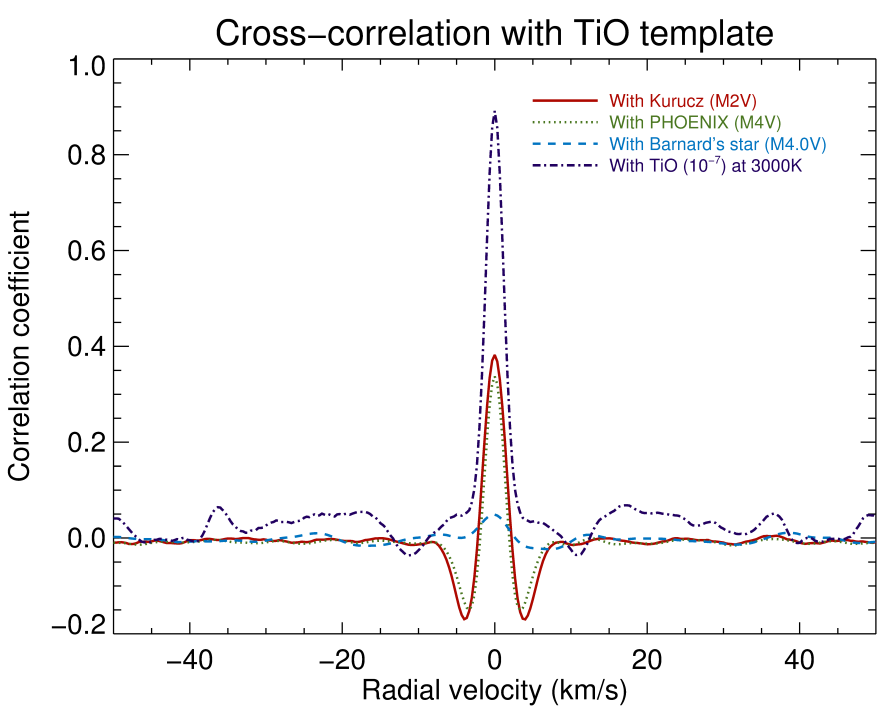

Fig. 6. Correlation of our $\mathrm{TiO}$ template (at $T \sim 1500 \mathrm{~K}$ ), as shown in Fig. 4, with the PHOENIX M4V model (green), the Kurucz model of GL411 (red), Barnard's star's optical spectrum (light blue) and the TiO template modelled at a temperature of $3000 \mathrm{~K}$ (dark blue). All modelled spectra correlate strongly with our TiO template, while Barnard's star's spectrum does not. The high peak correlation between the two templates at different temperatures suggests that the assumption of an inaccurate T-P profile has little effect on the templates.

spectrum. If the wavelength positions of the lines are inaccurate, the cross-correlation procedure may not add their contributions constructively. As shown below, this is a real concern.

\subsection{Evaluation of template spectra}

Because $\mathrm{TiO}$ absorption visibly dominates M-dwarf optical spectra, a high-resolution optical spectrum of Barnard's star can be used to test the accuracy of our TiO template. Their mutual correlation should be very clear.

Figure 6 shows the cross-correlation between our TiO template at different temperatures, the stellar atmosphere models and the HARPS-spectrum of Barnard's star. The TiO-bearing model of HD 209458b (VMR $=10^{-7}$, T-P profile as shown in Fig. 4) shows a correlation peak of 0.35 with the stellar atmosphere models. The cross-correlation between our TiO templates at $3000 \mathrm{~K}$ and $\sim 1500 \mathrm{~K}$ peaks at 0.9 . M-dwarf transmission spectra feature a host of other optical absorbers, which decrease the correlation between an M-dwarf spectrum and a pure-TiO template. We regard this as the main reason for the poor correlation between our $\mathrm{TiO}$ template and the stellar atmosphere models, rather than a mismatch of the T-P profile associated with the template.

In contrast to the stellar atmosphere models, a marginal correlation peak of only 0.1 is observed between the TiO template and the HARPS spectrum. From this we may conclude that our TiO-bearing model spectra do not accurately reproduce realworld $\mathrm{TiO}$ absorption at high resolution.

To test the extent to which stellar atmosphere models and real M-dwarf spectra match each other at high resolution, we cross-correlated Barnard's star's spectrum with the spectra of five other M dwarfs and with the PHOENIX M4V model. This is shown in Fig. 7. Regardless of differences in effective temperature, metallicity, and $\mathrm{S} / \mathrm{N}$, real $\mathrm{M}$-dwarf spectra correlate strongly with each other; with a peak of $>0.75$ in all cases.
Cross-correlation M-dwarf spectra and PHOENIX

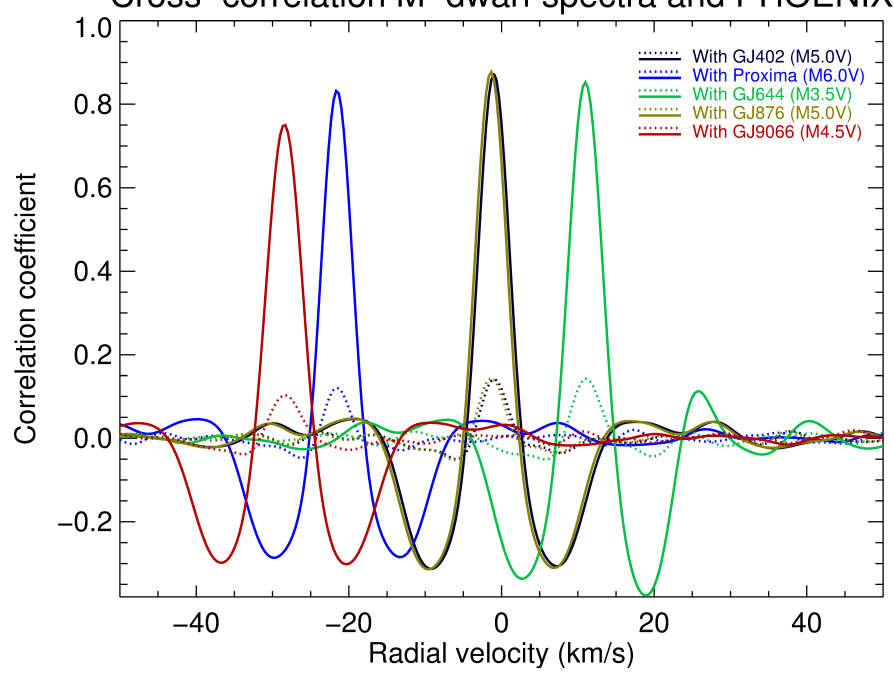

Fig. 7. Correlation of several M-dwarf spectra (see Table 2) with Barnard's star (solid lines) and with the PHOENIX M4V model (dashed lines). The cross-correlation procedure retrieves the Doppler shifts of the other M dwarfs, since we did not shift these to their own rest frames, for the sake of clarity. All M dwarfs correlate by more than 0.75 with Barnard's star, while correlation with the model absorption spectrum never exceeds 0.15 .

However, as is the case with the TiO template, the PHOENIX model correlates at less than 0.15 with all of the M-dwarf spectra. This strengthens our conclusion that the modelled spectrum of $\mathrm{TiO}$ is not representative of real $\mathrm{TiO}$. The high correlation between the TiO template and the PHOENIX and Kurucz models, suggests that the TiO line lists have a common source, and the same uncertainties in the line positions seem to have persisted in the works of both Plez (1998) and Schwenke (1998). A visual comparison of the models and the spectrum of Barnard's star, shows strong similarity between all three models at high resolution, but a severe mismatch with the HARPS data (Fig. 8).

Although the TiO models do not match the HARPS data well, our TiO template is able to partially retrieve the presence of TiO in the Barnard's star spectrum. The extent of the inaccuracies of the models, as shown in Fig. 8 near $530 \mathrm{~nm}$, raises the suspicion that the marginal correlation with Barnard's star is not due to the haphazard alignment of model absorption lines. Instead, it is possible that certain absorption bands are modelled more accurately than others. To test the hypothesis that inaccuracies are only associated with certain absorption bands only, we divide the available $130 \mathrm{~nm}$ range of our HARPS data into smaller sections and again cross-correlate our model of HD 209458b with each section separately. The result is shown in Fig. 9.

Over most of the wavelength range, our model does not correlate with the $\mathrm{TiO}$ spectrum. However above $630 \mathrm{~nm}$, the $\mathrm{TiO}$ template appears to match the data better, increasing in correlation toward longer wavelengths (a trend that may continue further toward the near infrared). The four sections shown in Fig. 9 correlate most at slightly different radial velocity shifts (few $\mathrm{km} \mathrm{s}^{-1}$ ), meaning that ensembles of lines are collectively offset. This specifically indicates inaccuracies in the calculated values of the energy levels of the TiO molecule. 


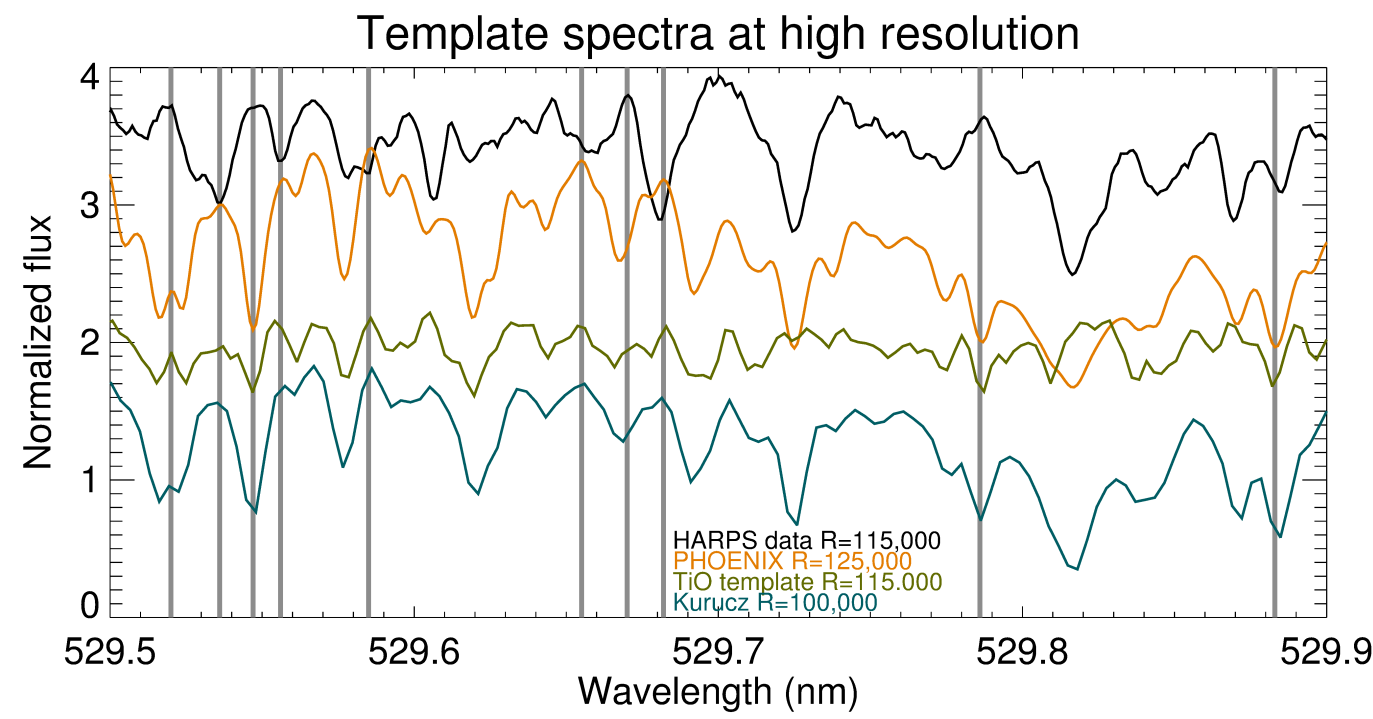

Fig. 8. Visual comparison of the three TiO-bearing model atmospheres and the HARPS-spectrum of Barnard's star. The spectra are vertically offset for clarity. Although some broad absorption lines of the stellar models seem to line up with the HARPS-data, most narrow-band features (the majority of them are due to $\mathrm{TiO}$ ) are clearly misplaced. Some obvious mismatches are indicated by the gray vertical lines.

\subsection{Alternative template spectra}

As shown above, the cross-correlation procedure is very sensitive to species with rich optical absorption spectra in the HDS residuals. As for TiO, the sensitivity reaches down to VMRs below the ppb level. Although we have also shown that the line lists that are the basis of our $\mathrm{TiO}$ (and others) model spectra display extensive inaccuracies, the template is able to partially retrieve TiO features from Barnard's star's optical spectrum at wavelengths longward of $\sim 640 \mathrm{~nm}$ and is thus to a certain extent useful for probing the atmosphere of HD 209458b for the presence of TiO. We therefore cross-correlate the residuals with the TiO template at a VMR of $10^{-7}$, using only wavelengths between $640 \mathrm{~nm}-682 \mathrm{~nm}$, the result of which is shown in the top row of Fig. 10.

Injecting the template into the residuals like before reveals a theoretical sensitivity of $7.6 \sigma$ (top right panel of Fig. 10). However, because the line list is only partially accurate at these wavelengths, this is only an upper limit: That is, if line list was perfect, the presence of TiO in the atmosphere of HD 209458b at a VMR of $10^{-7}$ would have been retrieved with a significance of $7.6 \sigma$. Though since the line list suffers from unknown inaccuracies, the true limiting VMR is not determined.

Earlier it was shown (Fig. 6) that if the temperature-pressure environment of the $\mathrm{TiO}$ template does not match that of the data, the correlation is not strongly affected. Therefore, although the temperature of the photosphere of Barnard's star is some $1500 \mathrm{~K}$ higher than the atmosphere of HD 209458b in the terminator region, the TiO absorption present in the spectra of $\mathrm{M}$ dwarfs may also be useful for identifying TiO absorption in exoplanet atmospheres at high resolution. As shown before (Fig. 7), the presence of noise in a cross-correlation template does not destroy the cross-correlation at high-resolution, and thus we propose to use the HARPS spectrum of Barnard's star as a template for crosscorrelation with the HDS data of HD 209458b.

Injecting the absorption spectrum of Barnard's star into the residuals of the HDS data does not provide a physically meaningful sensitivity. Although our template at $\sim 1500 \mathrm{~K}$ correlates significantly with the stellar atmosphere models, the absorption spectrum of an M dwarf is not a good model for the absorption spectrum of the upper atmosphere of HD 209458b during transit, because the underlying physical parameters are very different. In addition to the different temperature-pressure environments, the presence of other species in an M dwarf's photosphere and the different continuum level (HD 209458 is a solar type star) make it difficult to adjust Barnard's star's absorption spectrum to form a template from which parameters such as the limiting VMR can be derived as before. Extracting a physically meaningful $\mathrm{TiO}$ absorption spectrum from real-world absorption spectra of $\mathrm{M}$ dwarfs would require more work and is beyond the scope of this paper. For now, we only use Barnard's star's spectrum to qualitatively probe for the presence of $\mathrm{TiO}$ absorption in the HDS dataset. The resulting cross-correlation is shown in Fig. 10. Again, no significant correlation is detected.

\section{Conclusion}

We have probed the optical transmission spectrum of HD 209458b for TiO absorption using the time-dependent radial velocity of the planet, which can be spectrally resolved at high resolution. At first glance our null-result seems to in contrast with the possible detection of TiO by Désert et al. (2008) and to be in line with more recent observations of hot Jupiters and models. Injection of model TiO spectra into the data shows that our cross-correlation retrieval method is sensitive to VMR down to $10^{-10}$, and is thus theoretically capable of ruling out the presence of $\mathrm{TiO}$ at a stringent level.

However, this method relies on the accuracy of template spectra of $\mathrm{TiO}$ at resolutions of $R \sim 10^{5}$. Although such templates are available in the literature, we observe that the line databases used to synthesize these spectra are based on model calculations of the $\mathrm{TiO}$ molecule that display widespread inaccuracies. We infer that the energy levels of the TiO molecule are not determined well enough to accurately synthesize absorption spectra at these resolutions. Nonetheless, we have found that the TiO line list used to generate our template spectrum is accurate to some extent at wavelengths between $640 \mathrm{~nm}$ and $670 \mathrm{~nm}$ and that the spectra of $\mathrm{M}$ dwarfs can also serve as cross-correlation templates. However, using a high-resolution spectrum of Barnard's star and restricting our template to the 


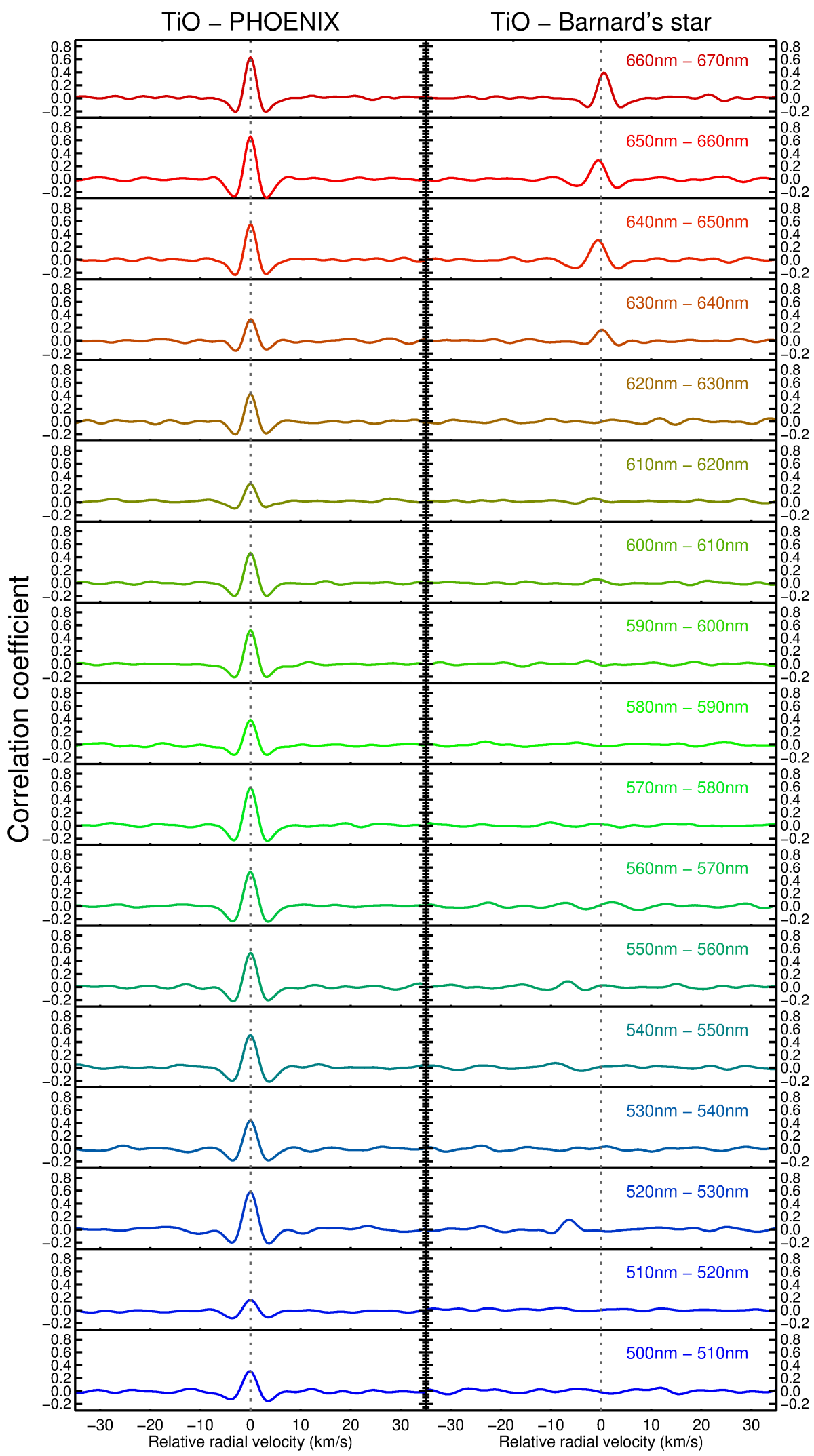

Fig. 9. Cross-correlation of sections of the $\mathrm{TiO}$ template with the PHOENIX model (left column) and with the Barnard's star spectrum (right column), at different wavelengths. The TiO template correlates strongly almost everywhere between $500 \mathrm{~nm}$ and $670 \mathrm{~nm}$, while the correlation between the template and the TiO-dominated spectrum of Barnard's star is absent across most of the wavelength range, and less than 0.4 in the best case. We also note that maximum correlation occurs at slightly different radial velocities (an extreme feature is observed between $520 \mathrm{~nm}$ and $530 \mathrm{~nm}$ ). This is explicitly indicative of wavelengthoffsets of entire TiO bands (and thus inaccuracies in the determination of the energy levels of the $\mathrm{TiO}$ molecule). aforementioned wavelength range do not result in a significant correlation. It would therefore seem that the presence of a high concentration of $\mathrm{TiO}$ in the upper atmosphere of HD 209458b in the terminator region is unlikely. However, owing to the uncertainties regarding the physical meaning of these template models, such a conclusion is tentative.
The lack of an accurate TiO line list is thus a critical hindrance to applying this retrieval technique to search for the presence of $\mathrm{TiO}$ in the optical transmission spectrum of HD 209458b. Also, since the TiO molecule is among the best characterized molecules in astrophysics, we suspect that the absorption spectra of less common and more complicated species are likely to 

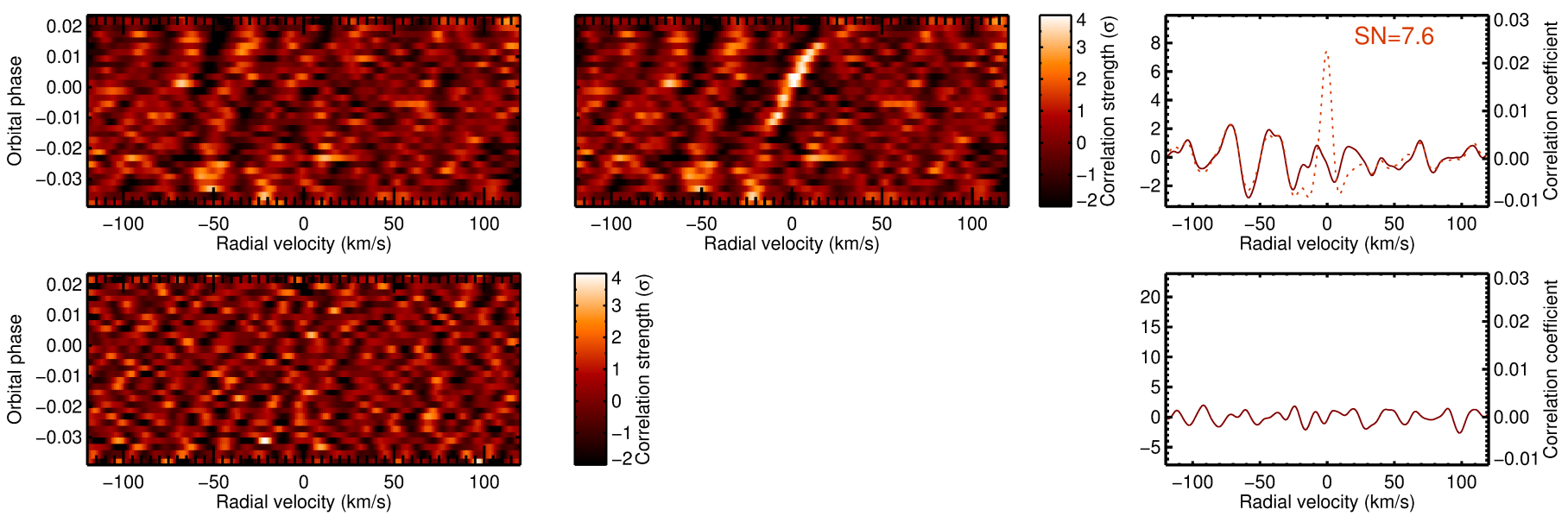

Fig. 10. Top panels: correlation coefficients for cross-correlation with the TiO template (VMR $=10^{-7}$ ) between $640 \mathrm{~nm}$ and $670 \mathrm{~nm}$, with and without injecting the template into the data prior to cross-correlation (middle and left panel, dashed and solid lines respectively). Restricting the wavelength range has reduced the $\mathrm{S} / \mathrm{N}$ at which this template is retrieved after injection by over $9 \sigma$ (compare to Fig. 5), but it is not clear to what extent persistent inaccuracies still adversely affect the sensitivity of this restricted template. Bottom row: correlation coefficients for crosscorrelation Barnard's star's optical spectrum. No enhanced correlation is observed. Given that the injection of the stellar absorption spectrum into the planetary transmission spectrum is not physically meaningful, the sensitivity of this non-detection can't straightforwardly be expressed in physical quantities like the limiting VMR.

be even less accurately characterized. Furthermore, inaccuracies in the calculations of line positions not only severely hinder the application of high-resolution retrieval techniques like the one employed in this work, but they also pervade widely used stellar atmosphere models. Modern-day stellar atmosphere codes apply highly advanced modelling techniques to poor line databases, which is over a decade old in the case of $\mathrm{TiO}$.

Therefore we conclude that an increasing community of observational and theoretical astronomers would benefit from an immediate and extensive effort to improve the quality of the absorption spectra of molecules like TiO, either through more sophisticated theoretical calculations or by means of direct measurements in the laboratory.

Acknowledgements. This work is part of the research programmes PEPSci and VICI 639.043.107, which are financed by the Netherlands Organisation for Scientific Research (NWO), and was also supported by the Leiden Observatory Huygens Fellowship. This work was performed in part under contract with the California Institute of Technology (Caltech)/Jet Propulsion Laboratory (JPL) funded by NASA through the Sagan Fellowship Program executed by the NASA Exoplanet Science Institute. Support for this work was provided in part by NASA through Hubble Fellowship grant HST-HF2-51336 awarded by the Space Telescope Science Institute. Also, this research has made use of the Exoplanet Orbit Database and the Exoplanet Data Explorer at exoplanets.org. Finally, we would like to thank Leigh Fetcher, Jaemin Lee and Patrick Irwin for providing the TiO line-list needed to perform this analysis.

\section{References}

Asplund, M., Grevesse, N., \& Jacques Sauval, A. 2006, Nucl. Phys. A, 777, 1 Bento, J., Wheatley, P. J., Copperwheat, C. M., et al. 2014, MNRAS, 437, 1511 Birkby, J. L., de Kok, R. J., Brogi, M., et al. 2013, MNRAS, 436, L35

Brogi, M., Snellen, I. A. G., de Kok, R. J., et al. 2012, Nature, 486, 502

Brogi, M., Snellen, I. A. G., de Kok, R. J., et al. 2013, ApJ, 767, 27

Brogi, M., de Kok, R. J., Birkby, J. L., Schwarz, H., \& Snellen, I. A. G. 2014, A\&A, 565, A124

Burrows, A., \& Orton, G. 2009, in Exoplanets, ed. S. Seager (University of Arizona Press) [arXiv: 0910.0248]

Burrows, A., Hubeny, I., Budaj, J., Knutson, H. A., \& Charbonneau, D. 2007, ApJ, 668, L171
Castelli, F., \& Kurucz, R. L. 2003, in Modelling of Stellar Atmospheres, eds. N. Piskunov, W. W. Weiss, \& D. F. Gray, IAU Symp., 210, 20

Crossfield, I. J. M., Knutson, H., Fortney, J., et al. 2012, ApJ, 752, 81

de Kok, R. J., Brogi, M., Snellen, I. A. G., et al. 2013, A\&A, 554, A82

Désert, J.-M., Vidal-Madjar, A., Lecavelier Des Etangs, A., et al. 2008, A\&A, 492, 585

Diamond-Lowe, H., Stevenson, K. B., Bean, J. L., Line, M. R., \& Fortney, J. J. 2014, ApJ, 796, 66

Fortney, J. J., Shabram, M., Showman, A. P., et al. 2010, ApJ, 709, 1396

Freedman, R. S., Marley, M. S., \& Lodders, K. 2008, ApJS, 174, 504

Gibson, N. P., Aigrain, S., Barstow, J. K., et al. 2013, MNRAS, 436, 2974

Hubeny, I., Burrows, A., \& Sudarsky, D. 2003, ApJ, 594, 1011

Huitson, C. M., Sing, D. K., Pont, F., et al. 2013, MNRAS, 434, 3252

Husser, T.-O., Wende-von Berg, S., Dreizler, S., et al. 2013, A\&A, 553, A6

Knutson, H. A., Charbonneau, D., Noyes, R. W., Brown, T. M., \& Gilliland, R. L. 2007, ApJ, 655, 564

Knutson, H. A., Charbonneau, D., Allen, L. E., Burrows, A., \& Megeath, S. T. 2008, ApJ, 673, 526

Lockwood, A. C., Johnson, J. A., Bender, C. F., et al. 2014, ApJ, 783, L29

Mazeh, T., Naef, D., Torres, G., et al. 2000, ApJ, 532, L55

Narita, N., Suto, Y., Winn, J. N., et al. 2005, PASJ, 57, 471

Nidever, D. L., Marcy, G. W., Butler, R. P., Fischer, D. A., \& Vogt, S. S. 2002, ApJS, 141, 503

Parmentier, V., Showman, A. P., \& Lian, Y. 2013, A\&A, 558, A91

Plez, B. 1998, A\&A, 337, 495

Portmann, R. W., \& Solomon, S. 2007, Geophys. Res. Lett., 34, 2813

Rodler, F., Lopez-Morales, M., \& Ribas, I. 2012, ApJ, 753, L25

Rodler, F., Kürster, M., \& Barnes, J. R. 2013, MNRAS, 432, 1980

Rowe, J. F., Matthews, J. M., Seager, S., et al. 2006, ApJ, 646, 1241

Schwenke, D. W. 1998, Faraday Discussions, 109, 321

Sing, D. K., Lecavelier des Etangs, A., Fortney, J. J., et al. 2013, MNRAS, 436, 2956

Snellen, I. A. G., Albrecht, S., de Mooij, E. J. W., \& Le Poole, R. S. 2008, A\&A, 487, 357

Snellen, I. A. G., de Kok, R. J., de Mooij, E. J. W., \& Albrecht, S. 2010, Nature, 465, 1049

Spiegel, D. S., Silverio, K., \& Burrows, A. 2009, in AAS/Division for Planetary Sciences Meeting Abstracts, 41, 68.03

Stevenson, K. B., Bean, J. L., Seifahrt, A., et al. 2014, AJ, 147, 161

Torres, G., Winn, J. N., \& Holman, M. J. 2008, ApJ, 677, 1324

Wright, J. T., Fakhouri, O., Marcy, G. W., et al. 2011, PASP, 123, 412

Zellem, R. T., Lewis, N. K., Knutson, H. A., et al. 2014, ApJ, 790, 53 